\title{
Dark current studies on a normal-conducting high-brightness very-high-frequency electron gun operating in continuous wave mode
}

\author{
R. Huang, ${ }^{2,1}$ D. Filippetto, ${ }^{1, *}$ C. F. Papadopoulos, ${ }^{1}$ H. Qian, ${ }^{1}$ F. Sannibale, ${ }^{1}$ and M. Zolotorev ${ }^{1}$ \\ ${ }^{1}$ Lawrence Berkeley National Laboratory, One Cyclotron Road, Berkeley, California 94720, USA \\ ${ }^{2}$ National Synchrotron Radiation Laboratory, University of Science and Technology of China, \\ Hefei, Anhui, 230029, China
}

(Received 8 October 2014; published 12 January 2015)

\begin{abstract}
We report on measurements and analysis of a field-emitted electron current in the very-high-frequency (VHF) gun, a room temperature rf gun operating at high field and continuous wave (CW) mode at the Lawrence Berkeley National Laboratory (LBNL). The VHF gun is the core of the Advanced Photo-injector Experiment (APEX) at LBNL, geared toward the development of an injector for driving the next generation of high average power x-ray free electron lasers. High accelerating fields at the cathode are necessary for the high-brightness performance of an electron gun. When coupled with $\mathrm{CW}$ operation, such fields can generate a significant amount of field-emitted electrons that can be transported downstream the accelerator forming the so-called "dark current." Elevated levels of a dark current can cause radiation damage, increase the heat load in the downstream cryogenic systems, and ultimately limit the overall performance and reliability of the facility. We performed systematic measurements that allowed us to characterize the field emission from the VHF gun, determine the location of the main emitters, and define an effective strategy to reduce and control the level of dark current at APEX. Furthermore, the energy spectra of isolated sources have been measured. A simple model for energy data analysis was developed that allows one to extract information on the emitter from a single energy distribution measurement.
\end{abstract}

DOI: 10.1103/PhysRevSTAB.18.013401

PACS numbers: 29.25.Bx, 29.20.Ej, 29.27.Bd

The remarkable improvement in electron beam brightness by modern photoguns is having a tremendous impact on the range of applications based on electron accelerators. High peak fields at the cathode are used in conjunction with ultrashort laser pulses focused on sub-mm spot sizes to generate a very high peak current and low emittance beams, which can be efficiently used for many different applications, such as driving fourth generation light sources [1], generation of $\mathrm{THz}$ radiation [2], probing structural dynamics at the subpicosecond time scale [3], or as injectors for laser-based compact accelerators [4].

After the successful demonstration of the x-ray free electron lasers, we are now on the verge of the next revolutionary step in ultrafast instrumentation that, through a leap in average flux by orders of magnitude (from $\sim 100 \mathrm{~Hz}$ to $\mathrm{MHz}$ repetition rates and beyond), will open the doors to new science, enabling low cross section experiments, and the study of more complex systems by the tremendous increase in signal-to-noise ratio. The electron photogun is one of the key subsystems required to allow for such a step forward, and several different technologies are being pursued in different laboratories

\section{*DFilippetto@1bl.gov}

Published by the American Physical Society under the terms of the Creative Commons Attribution 3.0 License. Further distribution of this work must maintain attribution to the author(s) and the published article's title, journal citation, and DOI. around the world to respond to that need [5]. MHz-class repetition rates require guns operating in direct current (dc) or in continuous wave (CW) modes. The simultaneous request of a high accelerating field and a $\mathrm{CW}$ or dc operation poses a significant number of technical challenges and trade-offs in the construction of such photoinjectors. One of the major limitations is represented by the amount of electrons that can be field emitted from the cavity walls. Such an unwanted electron current (dark current) generally occupies a large volume in phase space and most of the electrons get lost along the beam line in points with small transverse and/or longitudinal acceptance. Depending on the kinetic energy and on the incidence angle, electrons can be absorbed by the beam pipe, causing heat load on the beam line walls, generating secondary shower products, disturbing the electronic devices in the tunnel, altering measurements, and possibly damaging equipment.

Dark current emission is due to electron tunneling from surface asperities, where local fields, greatly enhanced by the presence of sharp extremities, can shrink the potential barrier to vacuum allowing for electron tunneling. Particulates and intrusions of extraneous elements that can locally lower the work function have also been found to be sources of relevant amounts of field-emitted electrons [6]. In both cases, the intensity of the dark current follows the well-known Fowler-Nordheim exponential dependence with respect to the applied external field [7]. In radio-frequency (rf) guns, 
the temporal structure of the dark current is affected by the sinusoidal behavior of the field, with bunches of fieldemitted electrons generated around each field amplitude maximum.

In $\mathrm{CW}$ operation, field emission can lead to a large amount of average unwanted current (up to milliamperes) that if transported along the beam line can limit or jeopardize the facility operation. Therefore, the maximum accelerating field at the cathode is generally set by a compromise between beam dynamics considerations (high brightness requires high fields) and the maximum acceptable level of dark current. The selection of different beam aspect ratios at the cathode can help in preserving the beam brightness in the presence of relatively low electric fields [8], but in general it is of paramount importance to characterize and define an effective strategy to reduce the dark current from the electron gun as much as possible.

In this paper, we report on the results of a systematic study of dark current emission from the VHF gun of the Advanced Photo-injector Experiment (APEX) at the Lawrence Berkeley National Laboratory (LBNL), a CW source of picosecond electron beams with measured accelerating electric fields at the cathode in excess of $20 \mathrm{MV} / \mathrm{m}$, the highest demonstrated so far for a high repetition rate electron source. The measurements allowed us to identify and characterize the main sources of dark current, and to define an effective multifront strategy for significantly reducing the dark current. The defined steps, which include advanced cleaning techniques and a passive removal of the field-emitted electrons, are described and will be tested at APEX. Also described is an active removal scheme using a fast kicker and a collimator.

\section{APEX LAYOUT}

APEX at LBNL has been conceived and developed to demonstrate the capability of a new concept rf gun, the VHF gun $[9,10]$, of delivering electron beams with quality required by $\mathrm{x}$-ray free electron laser (FEL) applications at
MHz-class repetition rates. APEX is staged in three phases, with the first, Phase- 0 , dedicated to the demonstration of the rf and vacuum performance of the VHF gun and to initiate the characterization of several different types of high quantum efficiency $(\mathrm{QE})$ photocathodes. This phase is now successfully completed and the results are reported elsewhere [11]. For the sake of the topic treated here it is worth remarking that the gun rf conditioning was carried out without detecting any breakdown-induced arc inside the gun cavity. Indeed the peak field is limited by the available rf power driving the cavity and by surface heating. The vacuum pressure after the bake-out reached less than $4 \times 10^{-11}$ Torr in absence of rf, and $8 \times 10^{-10}$ Torr during operation at the nominal power. Residual gas analysis measurements indicated that the residual gas is mainly hydrogen with partial pressures of $\mathrm{H}_{2} \mathrm{O}, \mathrm{CO}$, and $\mathrm{CO}_{2}$ never exceeding $10^{-12}$ Torr. In the subsequent stage of the project, Phase-I, a suite of electron beam diagnostics is added to the existing beam line to allow for the 6D phasespace characterization of the beam. Figure 1 shows the layout of Phase-I with its main subsystems in evidence, and Fig. 2 shows a cross section of the VHF gun. All beam line components, with the only exception of the buncher cavity, are presently installed. The installation of Phase-II, the last of the project, will be completed in the first part of 2015. In this final stage, the addition of a small linac will allow one to accelerate the beam at relativistic energies $(\sim 30 \mathrm{MeV})$ and characterize the beam brightness in a regime where space charge forces are small enough to allow for reliable measurements.

The majority of the measurements presented in this paper were performed using the Phase-I beam line configuration shown in Fig. 1. The three removable view-screen systems located along the beam line and in the spectrometer branch allow selecting between YAG (higher resolution) and $\mathrm{BeO}$ fluorescent screens. The charge per bunch is measured by two integrated current transformers (ICT by Bergoz Inc.), while the beam orbit is measured by beam position monitors (BPMs) of the stripline type. Actuators in the

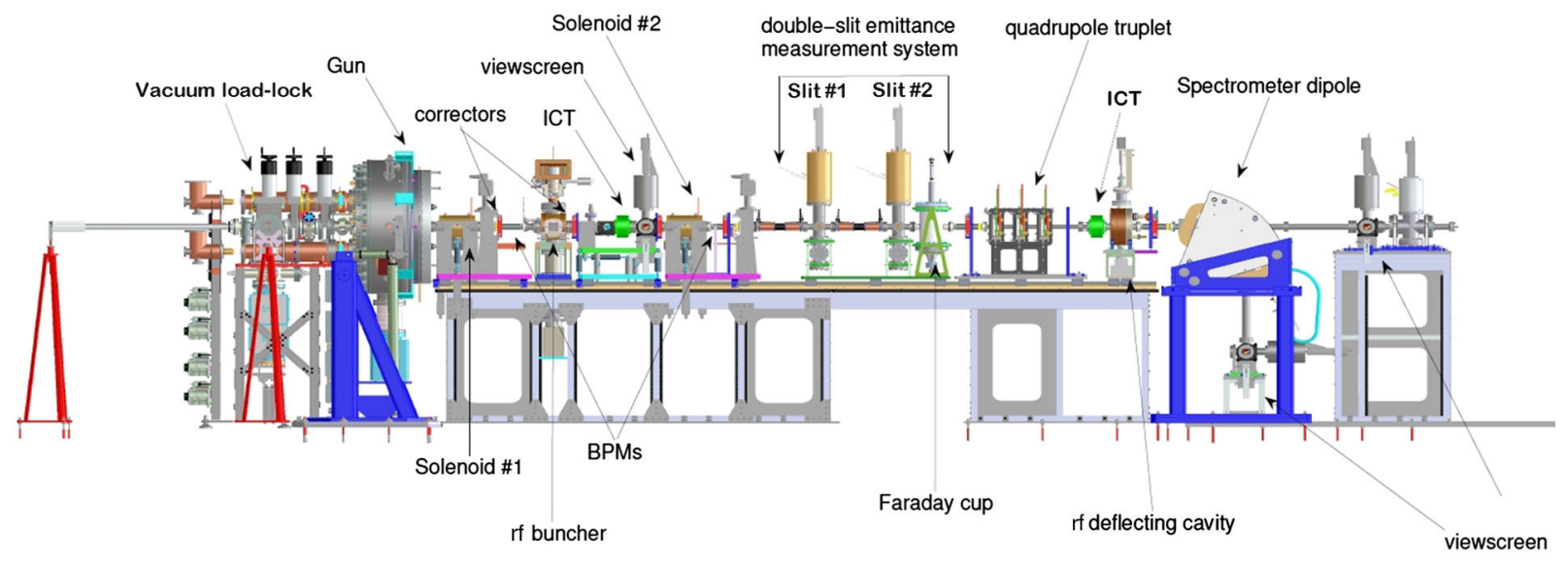

FIG. 1. Layout of the APEX Phase-I beam line. Although shown in the layout, the rf buncher is currently under construction. 


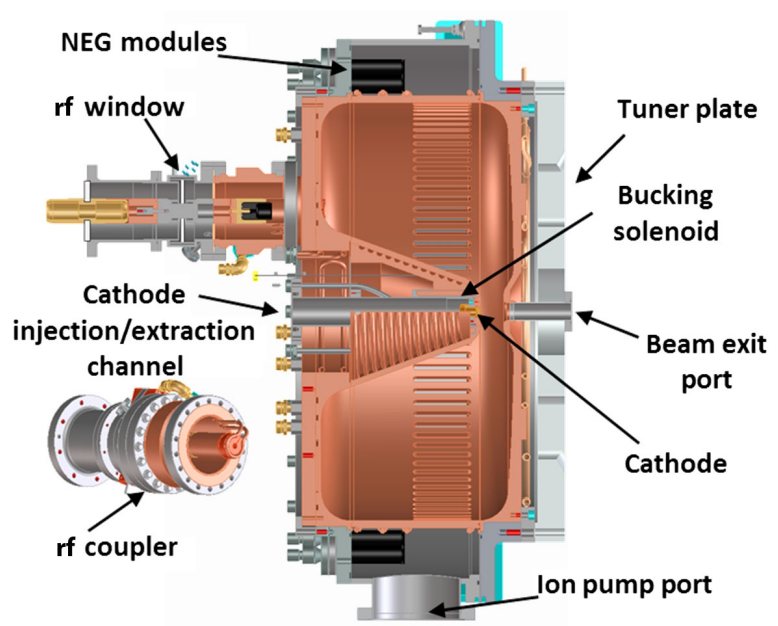

FIG. 2. CAD cross section of the VHF gun, with main components in evidence. The gun copper rf cavity resonates at $186 \mathrm{MHz}$ (7th subharmonic of $1.3 \mathrm{GHz}$ ) and operates in continuous wave mode accelerating beams at a nominal energy of $750 \mathrm{keV}$ with a gradient at the cathode of $\sim 19.5 \mathrm{MV} / \mathrm{m}$.

double-slit emittance meter "towers" allow one to select between horizontal or vertical slits, a fluorescent screen $(\mathrm{BeO})$, and an aperture that permits the unperturbed passage of the beam. Also, the Faraday cup downstream the second emittance meter tower is extractable.

\section{DARK CURRENT MEASUREMENTS, ANALYSIS, AND SIMULATIONS}

Dark current measurements in APEX included imaging of field emission sources located in the cathode area; measuring dark current versus accelerating field at the cathode (for a single emitter and integrated over all emitters); and measurements of the dark current energy spectrum. In this section we report the results and the analysis of such measurements.

\section{A. Sources of a dark current in the VHF gun}

In APEX the photoemitting material is deposited on a molybdenum plug that can be inserted into the gun by the vacuum load-lock system located in the rear side of the gun. When inserted, see Fig. 3, only the tip part of the plug is exposed to the rf fields in the gun. This part has a radius of $5 \mathrm{~mm}$ and is surrounded by the copper of the cavity nose.

By properly tuning solenoid 1 and 2, dark current electrons were used to create on the screen located in the slit 1 tower an image of the field emitters at the cathode. On the left side of Fig. 4 an example of those images is shown. Several field emitting points located with good accuracy along a ring are clearly visible. The image magnification was calibrated with the help of ASTRA simulations [12], revealing a ring radius of $\sim 5.3 \mathrm{~mm}$, implying the sources were located just outside the molybdenum plug, on the copper side surrounding the plug itself.

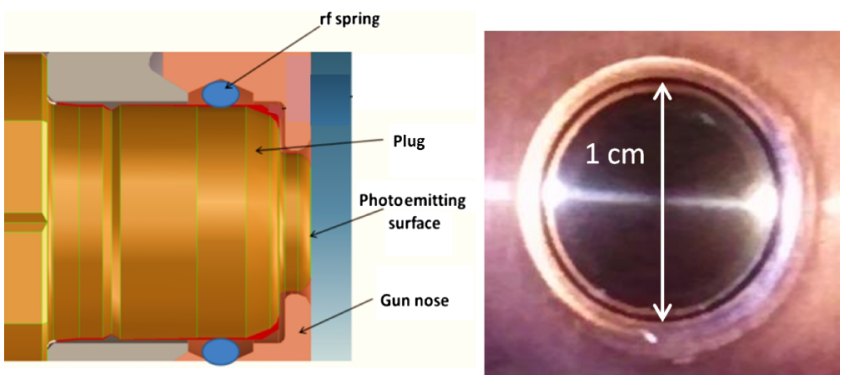

FIG. 3. Left: CAD side view of the molybdenum cathode plug inserted in the gun nose. Right: picture showing the plug tip inside the gun viewed from the beam exit pipe.
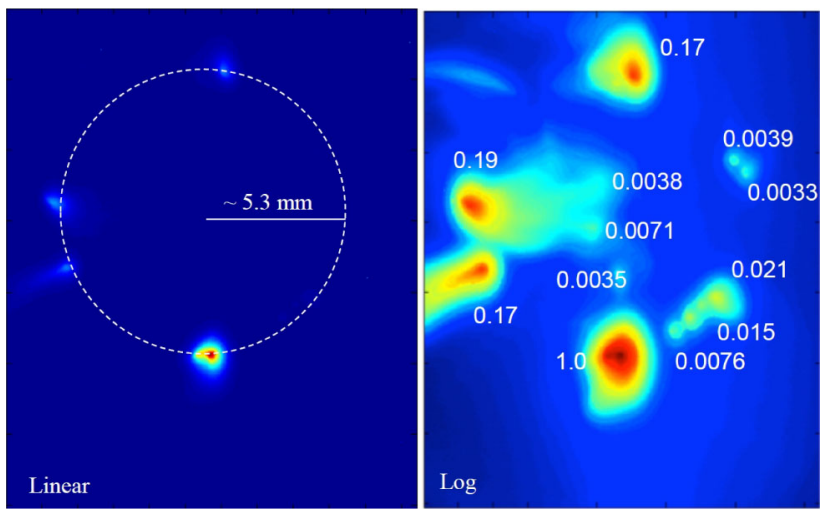

FIG. 4. Left: image of the field emitters in the cathode area. Right: the same image with the intensity plotted in logarithmic scale shows the relative intensity of the peaks.

Indeed, no image change was observed upon cathode plug rotation by $30^{\circ}$ via the load-lock system, confirming the hypothesis on the emitters location.

The right side of Fig. 4 shows the dark current in the same imaging conditions, but with a much higher dynamic range $\left(10^{6}\right)$ for the intensity, unveiling also the weakest sources. The figure intensity is now in logarithmic scale, and the relative weight of the individuals emitters is compared with the highest intensity emitter. The required dynamic range has been obtained by combining 13 individual images where the camera exposition duration was progressively varied from $100 \mu$ s to $100 \mathrm{~ms}$, while keeping a fixed camera gain.

\section{B. Dark current propagation along the beam line}

Using the information from the experimental observation above, it was possible to simulate the dark current propagation from the emitters to downstream of the beam line. We represented the dark current source by four field emitters laying on the $5.3 \mathrm{~mm}$ radius circle, and separated by $90^{\circ}$.

The temporal distribution was retrieved from the FowlerNordheim fit of the data (see Fig. 10) taken before Phase-0 


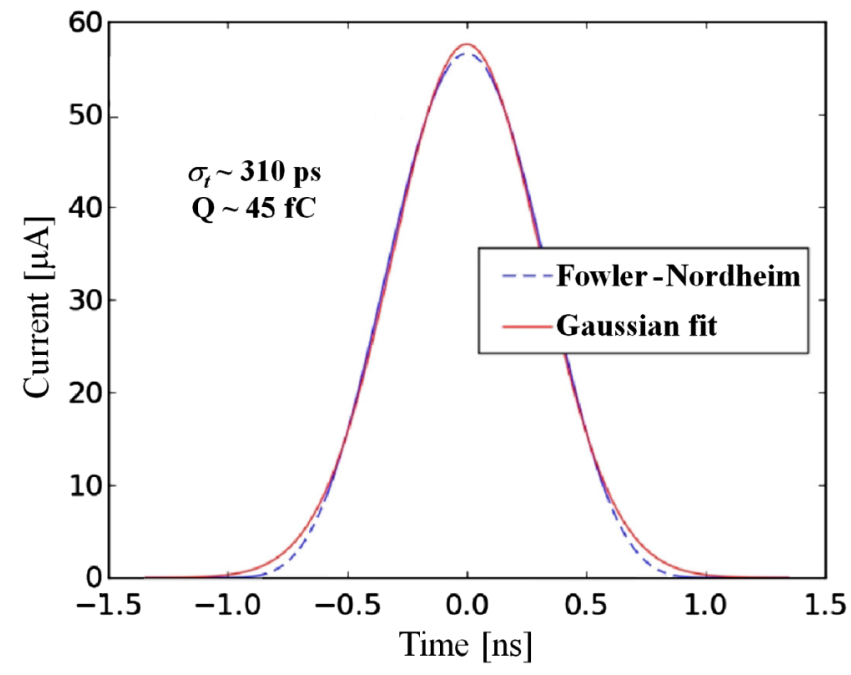

FIG. 5. Longitudinal distribution calculated using the FowlerNordheim fit in Fig. 10. Also shown is the Gaussian fit used for the simulations.

using a Faraday cup mounted directly at the exit flange of the gun. The nominal accelerating field of $19.5 \mathrm{MV} / \mathrm{m}$ was used, and for each gun rf phase the instantaneous dark current was calculated using the value from the fit in the figure. The resulting longitudinal distribution is shown in Fig. 5, together with the Gaussian fit approximation that was used in simulations.

For the longitudinal and transverse momenta, we used a rectangular distribution with a root mean square $(\mathrm{rms})$ value equal to the momentum correspondent to $E_{F} / \sqrt{12}$ (with $E_{F}$ the Fermi energy of the metal where the field emitters are located, $7 \mathrm{eV}$ for $\mathrm{Cu}$ in our case). This is a conservative approach, as the real electron energy distribution has to take into account the density of states and is therefore not rectangular. Estimates of total energy spread of emitted electrons using Ref. [13] showed for the real case a rms value smaller by a factor between 2 and 3 . In this conservative assumption, the thermal contribution $(\sim \mathrm{kT})$ due to the cathode operational temperature of $\sim 45^{\circ} \mathrm{C}$ is negligible. The use of a larger energy spread in simulation results in a larger beam size along the beam pipe. This was taken as the worst-case study for the passive collimation considerations in Sec. III A. Simulations showed that, regardless of any reasonable size assumed for the emitters, most particles were lost inside the gun and that no electron within such initial momentum distribution can be transported through the beam line at the slit 1 screen position. This scenario is explained by the strong defocusing kick that off-axis particles receive from the rf fields at the gun exit.

Single particle simulations also showed that the only way for the electrons to be transported downstream the beam line is for them to have a net transverse momentum radially directed towards the center of the cathode that can compensate for the rf kick; see Fig. 6. A further investigation of the field configuration in the cathode area showed that the orientation of the electric field in the interface area between the cathode plug and the surrounding copper, see Fig. 7, can indeed generate the radial momentum component required for transporting the electrons. It also appears that, because of the same field configuration, electrons from any field-emitter present on the edge of the molybdenum cathode plug would receive an initial momentum pointing outward, hitting the cavity walls or the beam pipe just outside the gun before the first solenoid. In Fig. 8, we plot the final offset of the beam at the exit of the gun (15 cm from the cathode) versus the initial offset of the beam at the cathode. We compare the evolution of the centroid in the case of an idealized 1D field not including the field distortions caused by the gap between the cavity wall and the cathode gap (red line), with the case of the 3D field calculated including the gap geometry. Electrons emitted at the edge of the cathode plug receive a

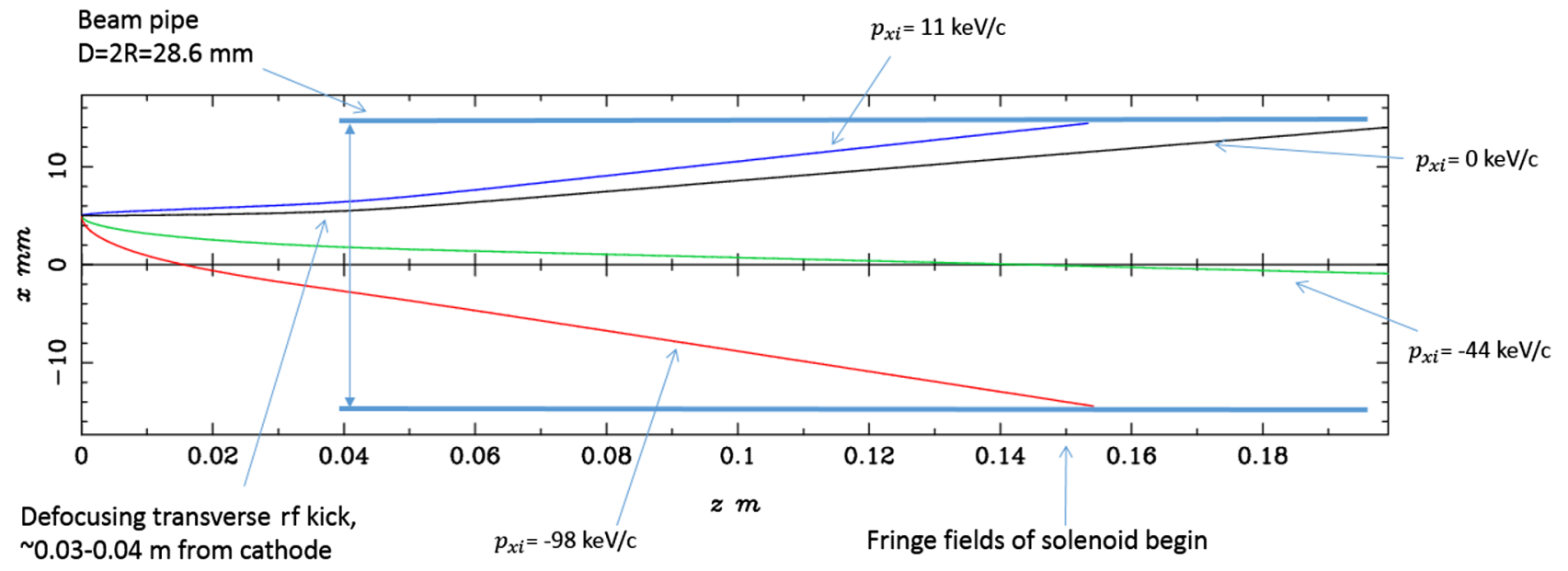

FIG. 6. Single particle tracking (ASTRA) showing the range of radially oriented transverse momenta required for electrons to be transmitted along the downstream beam line. 


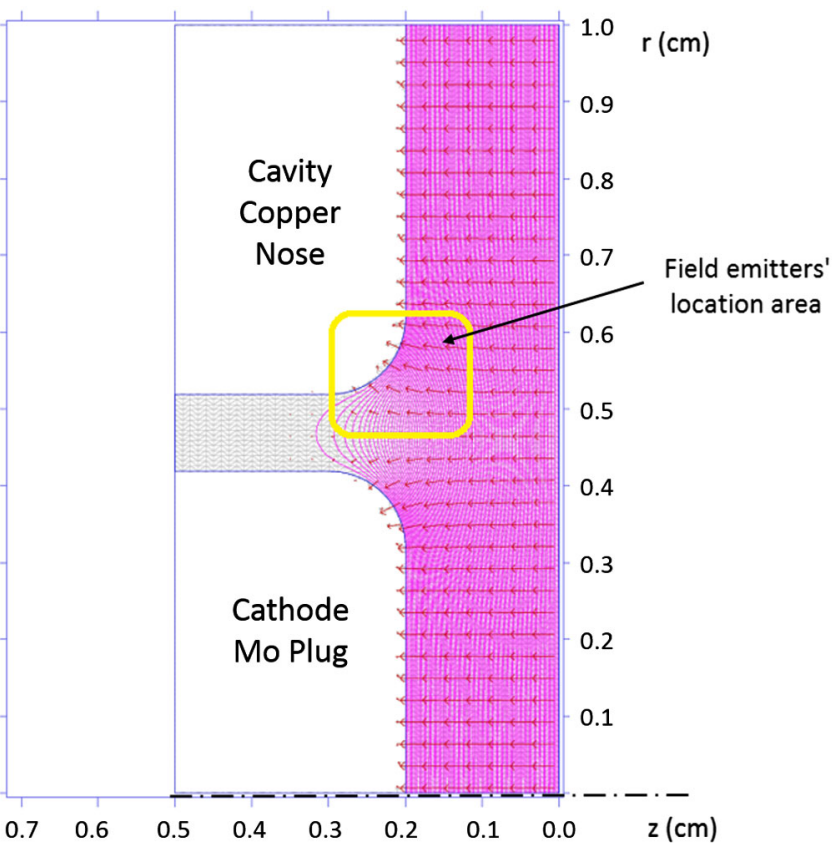

FIG. 7. Superfish simulation showing the electric field configuration in the interface area between the cathode plug and the gun cavity nose.

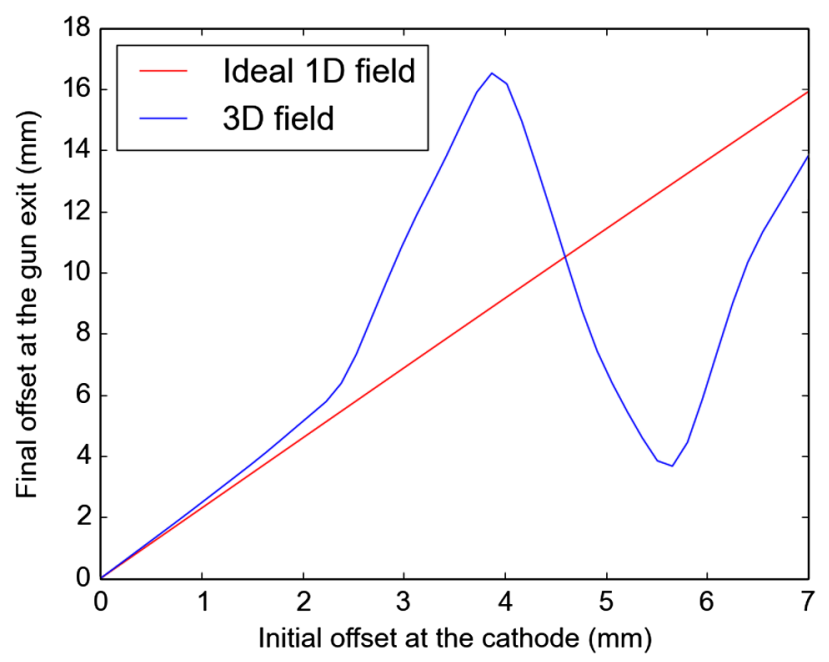

FIG. 8. Plot of beam offset at the cathode vs the final offset at the gun exit, for an ideal 1D field and a 3D field which includes the plug-cavity gap. Note that the first case is monotonic while the second case is not.

large defocusing kick (region between 2.5 and $3.5 \mathrm{~mm}$ ), while those emitted at the edge of the cavity copper nose are focused back close to the beam line axis (region between 3.5 and $5.5 \mathrm{~mm}$ ).

The average radial momentum value (within the range shown in Fig. 6) of electrons emitted by a particular emitter, depends on the precise radial position that emitter has along the rounded copper edge surrounding the cathode plug. Our measurements could not define such positions with the required accuracy, and in order to overcome this uncertainty in simulations, multiple cases with different initial transverse momentum distributions were simulated. The initial distributions were assumed to be Gaussian with the same rms value, correspondent to $E_{F} / \sqrt{12}$, but with a variable mean value, equidistributed over the range of transmitted radial momenta defined by the single particle simulations shown in Fig. 6. Subsequently, by comparing the similarity between the experimental image in Fig. 4 and the ones obtained from the simulations using the same magnet settings, it was possible to significantly reduce the range of possible radial momenta to values between -20 and $-40 \mathrm{keV} / \mathrm{c}$. Such values are compatible with the rf kick calculated using the expression in Ref. [14].

The knowledge of the field emitter's locations in the VHF gun allowed the definition and development of an effective strategy for reducing dark current. Details of the proposed methods are presented in Sec. III A.

\section{Quantifying dark current in APEX}

\section{Dark current scans}

In the previous section, it was shown that most of the field-emitted electrons generated at the gun are lost within the first few tens of $\mathrm{cm}$ along the beam line. Also, depending on the magnet settings, the transmitted fraction of such "surviving" electrons can show large variations, and quoting current values without specifying the specific beam line setting can be misleading. Also, the size of the Faraday cup detector with respect to the beam pipe diameter should be considered, as there may be a sizable fraction of dark current not being intercepted and therefore measured.

To account for this situation, we measured the dark current for different solenoid configurations, scanning for each setting a horizontal and a vertical corrector upstream the extractable Faraday cup while recording the dark current value measured by the cup itself. Figure 9 contains an example of such a measurement. The left side of the figure shows a comparison of the size of dark current main spot with the size of the instrument electrode. The image was taken on the screen of the slit 2 tower (just before the Faraday cup). The right side of the figure, shows the resulting scan data where the color of the individual points indicates the dark current measured for each couple of values of the correctors. The presence of a flat-top area in the figure confirms that the dark current spot was smaller than the Faraday cup electrode. Given the high dynamic range of the instrument we were able to measure haloes around the main spot, which show values always below the percent level (the blue area in the right picture). The measurement of Fig. 9, in which a total dark current of $500 \mathrm{nA}$ was recorded, was performed with an accelerating field in the cathode of $20.3 \mathrm{MV} / \mathrm{m}(\sim 780 \mathrm{keV})$ and with solenoid 1 and 2 optimized for emittance compensation [15] of a $100 \mathrm{pC}$ photoemitted bunch and for the 

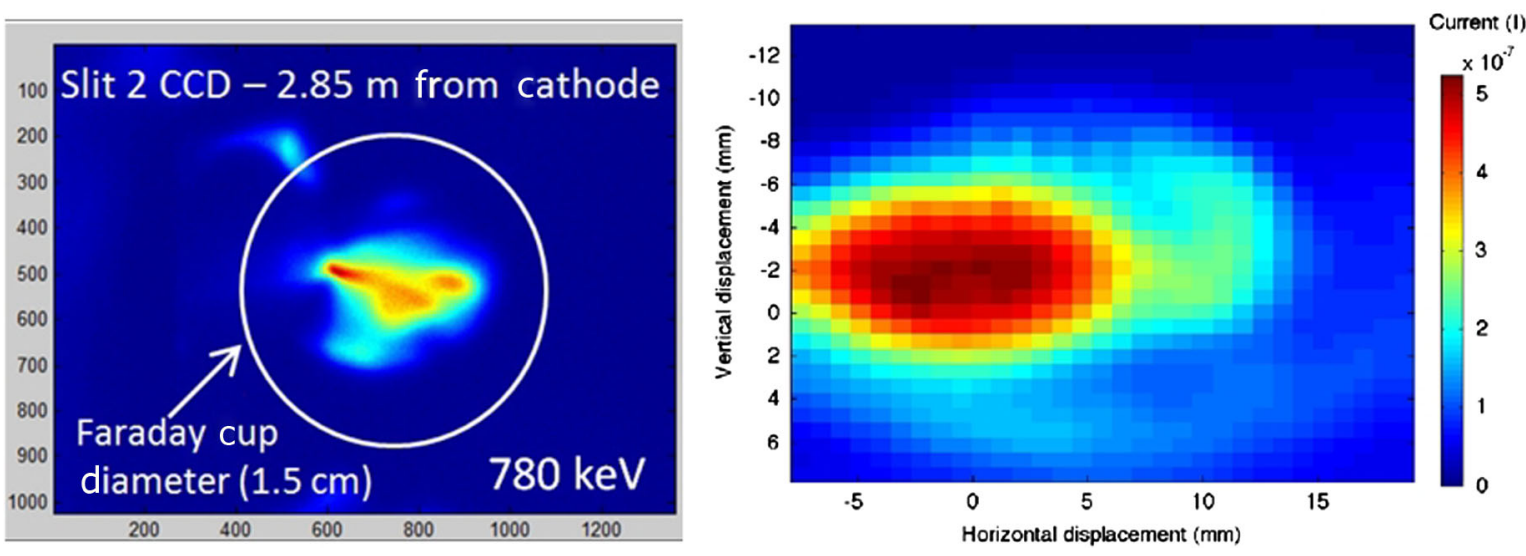

FIG. 9. Left: dark current focused on the screen on slit 2 right upstream the extractable Faraday cup (horizontal and vertical scales are in pixels). Right: example of dark current measurement at the Faraday cups by scanning horizontal and vertical steering coils.

compression required to operate in a typical x-ray FEL. Such a result holds also for different charges per bunch because simulations showed that in APEX, the optimal field in the first solenoid depends only weakly on the bunch charge. Indeed, going from 10 to $300 \mathrm{pC}$ per bunch requires field variations in this magnet of less than $10 \%$ [16]. Several additional scans with the same field at the cathode but for different solenoid settings were also performed, and the maximum transmitted dark current value of $\sim 2.5 \mu \mathrm{A}$ was measured when the solenoids were set for cathode imaging on the screen at second slit tower.

\section{Measurements of dark current vs electric field at the cathode and Fowler-Nordheim analysis}

A common procedure for field emission characterization is to plot the measured dark current as a function of the externally applied field, and then use the Fowler-Nordheim model to fit the data. We performed several of such scans which are reported and discussed in this section. During the initial part of the gun commissioning before the installation

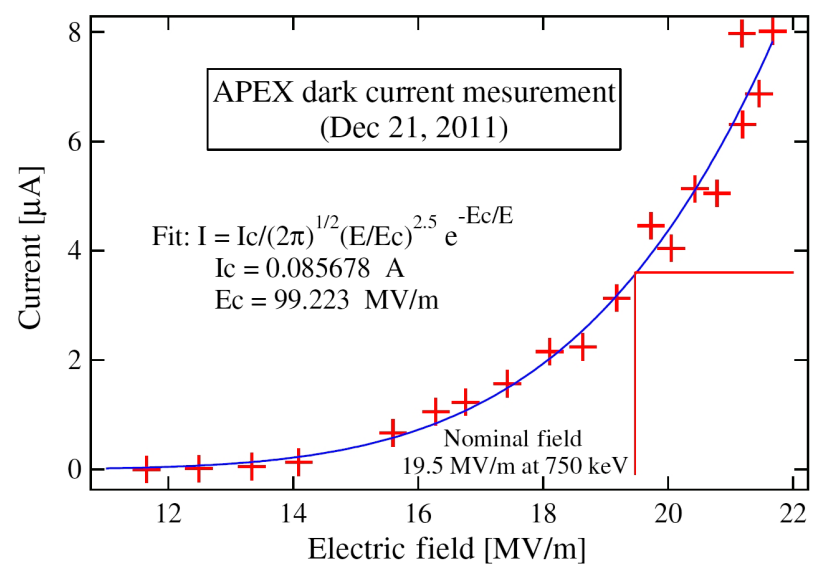

FIG. 10. Dark current vs electric field at the cathode measured by a Faraday cup directly attached to the gun beam exit pipe. A Fowler-Nordheim fit is also shown. of the APEX beam line, a Faraday cup was attached directly to the exit flange of the gun, maximizing the amount of electrons collected, and the dark current was measured as a function of the field. The results are reported in Fig. 10 and, as explained in Sec. II B, a fit of the data was used for retrieving the longitudinal distribution of field emitted electrons. After the installation of the beam line, dark current scans for fields at the cathode ranging from $\sim 16$ to $20 \mathrm{MV} / \mathrm{m}$ (equivalent to beam energies from $\sim 610$ to $780 \mathrm{keV}$ ) were performed using the corrector scan technique described in the previous section. The Faraday cup detector in this case was installed at about $3.2 \mathrm{~m}$ from the cathode plane, where a significant fraction of the dark current has been already lost along the first meters of the beam line vacuum chamber. Figure 11 shows the results obtained and the related FowlerNordheim fit. For the VHF gun nominal accelerating field of $19.5 \mathrm{MV} / \mathrm{m}, \sim 350 \mathrm{nA}$ were measured. Initial studies [17] indicate that such a value is already sufficiently low to operate in a FEL scheme using a CW superconducting linac without the risk of dark current-induced quenching in the

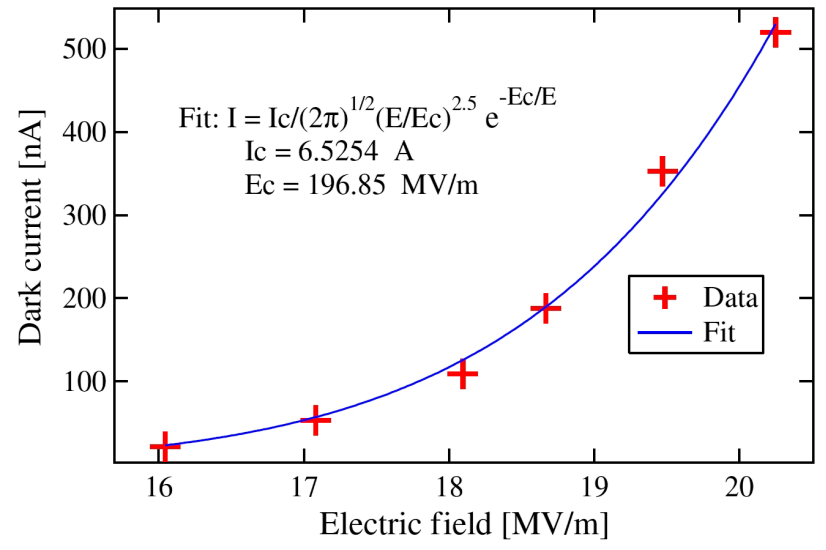

FIG. 11. Dark current vs electric field at the cathode. Also visible is the Fowler-Nordheim fit. 
linac, or degradation by radiation of the performance of the permanent magnets in the FEL undulators.

Studies of dark current emission in de [18], normalconducting rf [19], and super-conducting rf environments [20] have shown that the functional form of the FowlerNordheim formula, given in Eq. (1), is a good model for dark current emission in electron guns:

$$
\begin{aligned}
I_{\mathrm{FN}}= & \frac{1.54 \times 10^{-6} \times 10^{4.52 / \sqrt{\phi}} \times A_{e} \times \beta^{2} E^{2}}{\phi} \\
& \times \exp \left(-\frac{6.53 \times 10^{9} \phi^{1.5}}{\beta E}\right) .
\end{aligned}
$$

The Fowler-Nordheim formula above includes a number of parameters that are dependent on the model assumed for field emission as well as the detailed chemical composition of the emitter sites. In particular, $A_{e}$ refers to the area of the emitting site, $\phi$ refers to the work function of the emitting material, and $\beta$ is a local field enhancement factor that depends on the emitter geometry. The continuous usage of this theory is based on the fact that measurements of a dark current while increasing the applied field show an experimental relationship of the form $I_{\mathrm{dc}}=C_{1} E^{2} \exp \left(-C_{2} / E\right)$ where $I_{\mathrm{dc}}$ is the dark current and $E$ is the applied electric field. The fitting parameters $C_{1}$ and $C_{2}$ are related to the physically meaningful quantities discussed.

Traditionally, the fit has been done assuming a constant work function $\phi$ for the material, and fitting the values for the emitter area $A_{e}$ and the field enhancement factor $\beta$. This approach has been criticized in the literature as giving artificially low values for $A_{e}$ and high values for $\beta[18,21]$. In addition to this, detailed studies of emission sites in dc and superconducting rf high voltage environments [6] have shown that the field emitters are usually associated with unknown impurities of various chemical compositions that can present different work function values. Finally, it is known that surface effects such as cesiation may reduce the work function to lower and unknown values, making it reasonable to assume that the work function used in Fowler-Nordheim fits may not be the one of the substrate material. Indeed, unless a very detailed study and chemical analysis of field emitters is performed, treating $\phi$ as a fit parameter could represent the best approach.

For this, a model-agnostic approach for using the Fowler-Nordheim formula is to use the form

$$
I_{\mathrm{FN}}=I_{c} \frac{E^{2}}{E_{c}^{2}} \exp \left(-\frac{E_{c}}{E}\right) .
$$

The characteristic current and gradient, $I_{c}$ and $E_{c}$, include the physically meaningful fitting parameters discussed before, as shown in Eqs. (3) and (4):

$$
E_{c}=6.53 \times 10^{9} \frac{\phi^{1.5}}{\beta},
$$

$I_{c}=65.67 \times 10^{-6} \times 10^{4.52 / \sqrt{\phi}} \times A_{e}\left[n m^{2}\right] \times \phi^{2}$.

The physical meaning of the values $E_{c}$ and $I_{c}$ can be explained as follows. At a characteristic gradient $E_{c}$, typically much larger than the operating field $E_{0}$, the total emitted dark current would be $I=I_{c}$. Hence, $E_{c}$ and $I_{c}$ define a meaningful figure of merit for assessing the cavity dark current performances, higher $E_{c}$ and lower $I_{c}$ values implying lower electron field emission for a given accelerating field.

Following [19], in the case of dark current measured for a full rf period, we should include the effect of integrating, i.e. we write,

$$
\bar{I}_{\mathrm{FN}}=\frac{1}{T} \int_{-T / 2}^{T / 2} I_{\mathrm{FN}} d t
$$

Under the approximation that $E=E_{0} \cos (\omega t) \simeq$ $E_{0}\left(1-\omega^{2} t^{2} / 2\right)$ with $E_{0} \ll E_{c}$, the time integrated Fowler-Nordheim current becomes

$$
\bar{I}_{\mathrm{FN}}=\frac{1}{\sqrt{2 \pi}} I_{c}\left(\frac{E_{0}}{E_{c}}\right)^{2.5} \exp \left(-\frac{E_{c}}{E_{0}}\right) .
$$

The last equation has been used for fitting the data in Figs. 10, 11, and 14. Each plot reports the relative values of $E_{c}$ and $I_{c}$ found. The growth of the field-emitted current with the applied field is solely determined by the value of $E_{c}$, while $I_{c}$ plays a role in determining the absolute value of the current. In the particular case in which the number of sources does not change and the loss of electrons can be considered homogeneous among the sources, different machine settings and/or different measurement distances from the cathode plane only affect the fraction of the

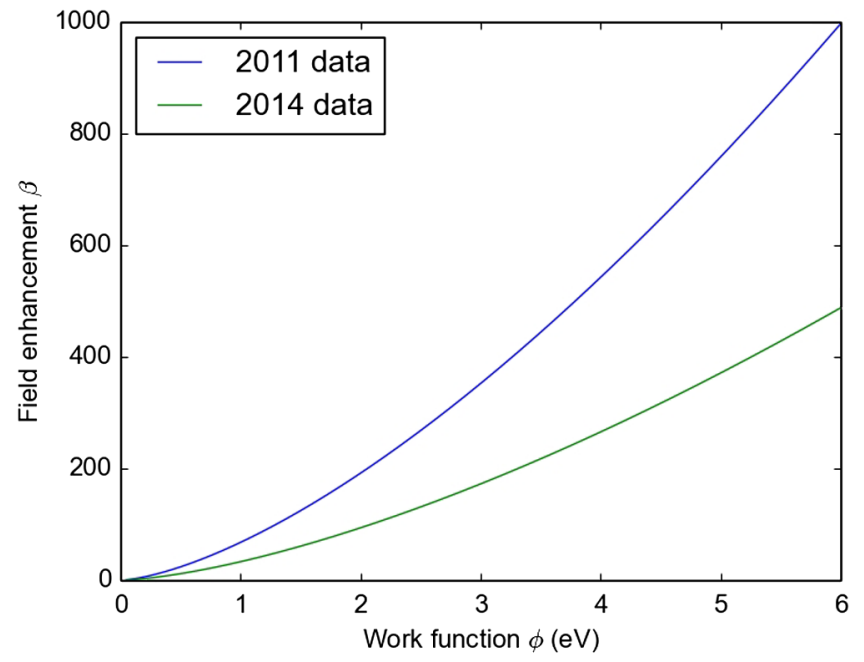

FIG. 12. $\phi$ vs $\beta$ plot from fitting the Fowler-Nordheim formula. A measurement of the emitter work function would yield the field enhancement due to the emitter shape and vice versa. 


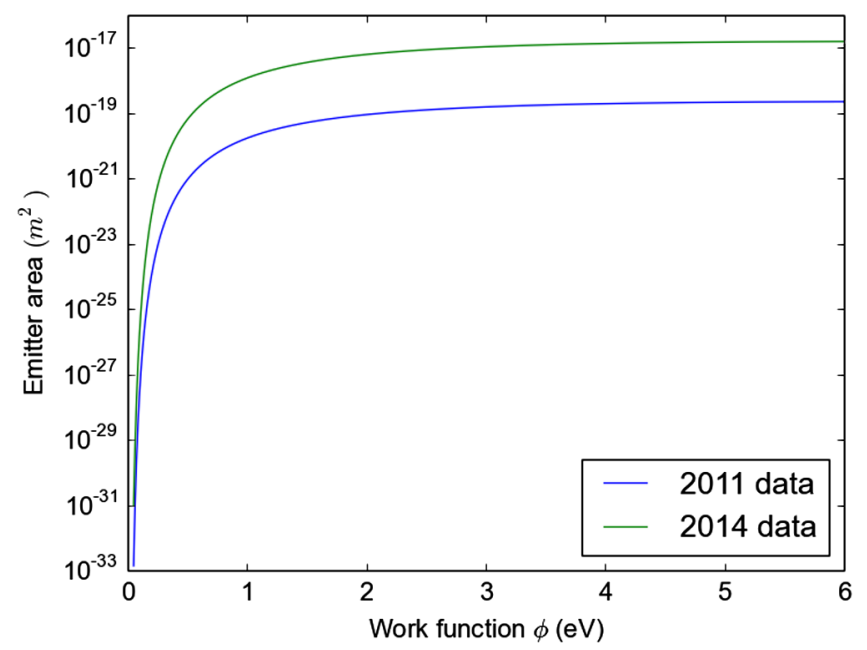

FIG. 13. $A_{e}$ vs $\phi$ plot from fitting the Fowler-Nordheim formula. A measurement of the emitter work function would yield the field enhancement and the area of the emitter.

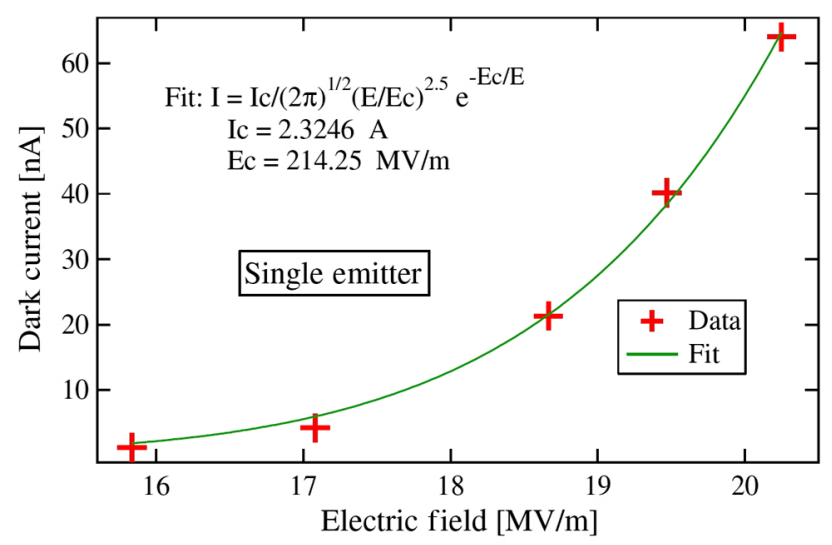

FIG. 14. Dark current vs electric field at the cathode for the single emitter in the top part of the circle of Fig. 4 (the one with relative weight of 0.17 ). Also visible is the Fowler-Nordheim fit.

current collected, i.e. $I_{c}$, without affecting its relative variation with the field (constant $E_{c}$ ). The measurements shown in Figs. 10 and 11 show a substantially different value of $E_{c}$. The former data set was collected at the beginning of the gun commissioning (December 2011) while the latter only in 2014, suggesting dark current reduction with time and operation.

Once the value of $E_{c}$ is found from the fit, the relation between the emitter's work function $\phi$ and the field enhancement factor $\beta$ (shape dependent) can be derived using Eq. (3). Figure 12 shows the $\phi, \beta$ plots associated with the data in Figs. 10 and 11 . For each $(\beta, \phi)$ only one value of $A_{e}$ is allowed. Figure 13 shows the curves of $A_{e}$ as a function of $\phi$ for the two cases of Figs. 10 and 11. The emitter area enters in the dark current formula as a scaling factor, hence the difference in measurement collection efficiencies.
By taking advantage of the spatial separation between different field emitters, we were able to characterize the field emission from a single emitter. The spot in the top part of the circle in Fig. 4 (relative weight of 0.17) was selected because, to the extent of our imaging system spatial resolution, it appears to be actually composed by a single emitting source. The current for different field values at the cathode was estimated by measuring the intensity of its peak in the CCD camera image. The peak value was then converted in an actual current by using the total current from all emitters as measured by the Faraday cup, and the relative weight information shown in Fig. 4. The results are shown in Fig. 14 with the related Fowler-Nordheim fit.

\section{Dark current energy distribution analysis}

In rf guns using $\mathrm{GHz}$ frequencies for acceleration ( $\mathrm{L}$ or $\mathrm{S}$ band), a significant phase slippage of the electron beam with respect to the accelerating field takes place during the transport in the gun. Hence in most cases the injection phase of the electron beam is far away from the on-crest phase (corresponding to the maximum field at the cathode), when most of the dark current is emitted. In the APEX gun case, because of the low frequency, the phase slippage is minimal (about $5 \mathrm{rf}$ degrees), and the beam is injected very close to the peak of the field. Thus photobeam and dark current kinetic energies are extremely close (see Fig. 16), and the same beam line settings can be used to transport both beams at the spectrometer screen (see Fig. 1) for energy measurements.

Because typical Fowler-Nordheim plots use spatially integrated dark current values, the measured current is usually due to few hot spots on the rf gun cavity wall. For APEX, this is clearly shown in Fig. 4, where the cathode imaging reveals the spatial separation between different field emitters but also different charge weights, determined by the source geometry and local work function. Such parameters in turn affect the temporal distribution of the emitted current and, consequently, its energy distribution. In Fig. 15, we show an example of high resolution measurement of the dark current energy profile with the maximum energy at $605 \mathrm{keV}$. By tuning the beam line, one single field emitter was isolated and transported to the

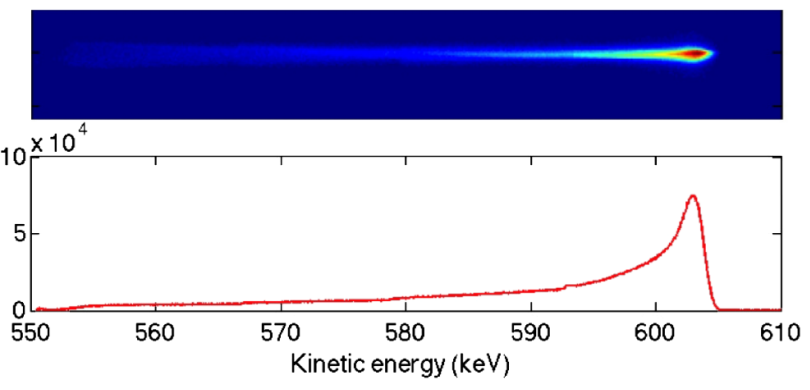

FIG. 15. Energy profile of single field emitter in the VHF gun. 


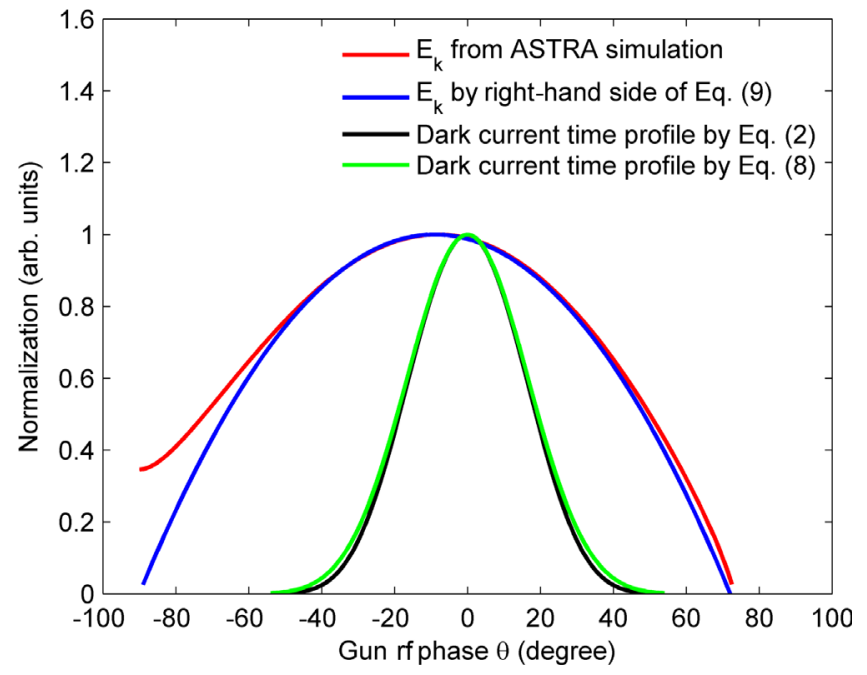

FIG. 16. Dark current energy and intensity as a function of rf phase in the APEX gun, in the case of $E_{0}=20 \mathrm{MV} / \mathrm{m}$ and $E_{c}=214 \mathrm{MV} / \mathrm{m}$.

spectrometer screen, and the quadrupole triplet was used for maximizing the energy resolution. In order to fit the data we used the Fowler-Nordheim formula for the instantaneous current [Eq. (1)] to derive a model for the dark current energy spectrum.

Considering a single field emission point in the rf gun, its surface electric field can be expressed as

$$
E(\theta)=E_{0} \cos \theta \approx E_{0}\left(1-\frac{\theta^{2}}{2}\right)
$$

where $E_{0}$ is the peak field in the gun, and $\theta$ is the $\mathrm{rf}$ emission phase. By combining the above equation with Eq. (2) and keeping terms up to the second order, the field emission current as a function of rf phase is found:

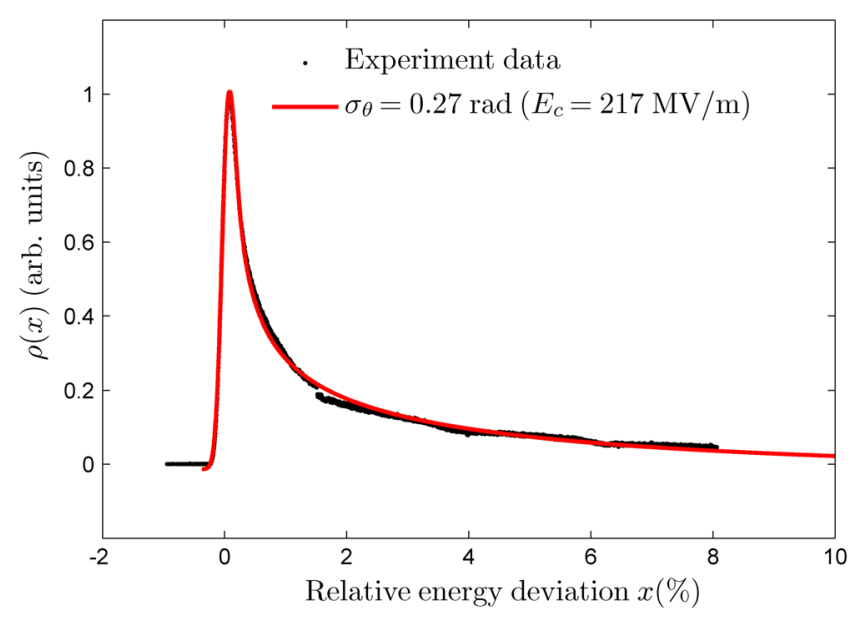

FIG. 17. Least square fit of the measured single emitter energy profile with the model of Eq. (13).

$$
I_{F}(\theta) \approx I_{0}\left(1-\theta^{2}\right) \exp \left(-\frac{\theta^{2}}{2 \sigma_{\theta}^{2}}\right)
$$

where $I_{0}=I_{c}\left(\frac{E_{0}}{E_{c}}\right)^{2} \exp \left(-\frac{E_{c}}{E_{0}}\right), \quad \sigma_{\theta}=\sqrt{\frac{E_{0}}{E_{c}}}$. Figure 16 shows an example of time profile derived from the Fowler-Nordheim model, by using the parameters derived from measurements in Fig. $14\left(E_{0}=20 \mathrm{MV} / \mathrm{m}\right.$, $E_{c}=214 \mathrm{MV} / \mathrm{m}, \sigma_{\theta}=0.3 \mathrm{rad}=17.6^{\circ}$, i.e $260 \mathrm{ps}$ ). Given the small phase slippage in the gun, the beam energy as a function of rf phase can be approximated as

$$
{ }_{E} k(\theta)=E_{\max } \cos \left(\theta+\theta_{0}\right) \approx E_{\max }\left(1-\frac{\left(\theta+\theta_{0}\right)^{2}}{2}\right) .
$$

In Fig. 16 we fit the energy curve produced by beam tracking simulations with Eq. (9) $\left(E_{\max }=750 \mathrm{keV}\right)$, finding $\theta_{0}=9^{\circ}$.

Combining Eqs. (8) and (9), the dark current energy spectra can be expressed as

$$
\begin{aligned}
\rho(x) \propto & \frac{1-\left(\sqrt{2 x}-\theta_{0}\right)^{2}}{\sqrt{2 x}} \exp \left(-\frac{\left(\sqrt{2 x}-\theta_{0}\right)^{2}}{2 \sigma_{\theta}^{2}}\right) \\
& +\frac{1-\left(\sqrt{2 x}+\theta_{0}\right)^{2}}{\sqrt{2 x}} \exp \left(-\frac{\left(\sqrt{2 x}+\theta_{0}\right)^{2}}{2 \sigma_{\theta}^{2}}\right),
\end{aligned}
$$

where $x=\frac{E_{\max }-E_{k}}{E_{\max }}$. Once $\theta_{0}$ and $\sigma_{\theta}$ are defined, the dark current spectra are fixed. The above formula can be simplified in two cases: (1) when $\theta_{0} \approx 0$,

$$
\rho(x) \propto \frac{1-2 x}{\sqrt{2 x}} \exp \left(-\frac{x}{\sigma_{\theta}^{2}}\right)
$$

and (2) when $-2 \sigma_{\theta}+\theta_{0}>0$,

$$
\rho(x) \propto \frac{1-\left(\sqrt{2 x}-\theta_{0}\right)^{2}}{\sqrt{2 x}} \exp \left(-\frac{\left(\sqrt{2 x}-\theta_{0}\right)^{2}}{2 \sigma_{\theta}^{2}}\right) .
$$

The energy distribution measured at the screen is the convolution between electron energy distribution and the spectrometer resolution, set by the beam transverse emittance:

$$
\rho_{\text {measured }}\left(\delta^{\prime}\right) \propto \int_{0}^{\infty} \rho(\delta) \mathrm{e}^{-\frac{\left(\delta^{\prime}-\delta\right)^{2}}{2 \delta_{\varepsilon}^{2}}} \mathrm{~d} \delta+\rho_{\text {floor }}
$$

where $\delta_{\varepsilon}$ is the rms energy resolution dilution at dipole screen caused by the finite transverse emittance, and $\rho_{\text {floor }}$ is the artificial energy density contribution from background noise, such as environment light, stray $\mathrm{x}$ rays and so on. A Gaussian transverse beam distribution was assumed.

When Eq. (13) is used to fit the energy profile from Fig. 15, the parameters extracted from the fit (reported in 
the picture) are in very good agreement with those retrieved by collecting dark current values as a function of the gun electric field.

Indeed, the time (energy) distribution of a field-emitted current contains the same amount of information as the time-integrated current dependence on the electric field. Such an experimental technique does not require one to scan the accelerating field, and can indeed be used as a fast alternative tool for field emission studies. It is worth noting that by calibrating the intensity of the spectrometer (vertical axis in Fig. 17) it would, in principle, be possible to extract also $I_{c}$ from the fit.

\section{DARK CURRENT MITIGATION METHODS}

The measurements described in the previous sections report dark current values for the VHF gun already compatible with the operation in injector schemes using superconducting $\mathrm{rf}$ in the $\mathrm{CW}$ mode. Nevertheless, the outcomes of such a measurement campaign paved the way for the definition of a multiple-step strategy to further reduce the amount of produced and transported dark current.

The use of experimentally tested cleaning techniques such as dry ice [22] or ethanol rinsing [23] applied to the gun cavity can drastically reduce the number of particulates and hence of potential field emitters. Dry ice cleaning of the cathode area of the VHF gun, where the dark current sources are located, will be tested in APEX in the near future.

The peculiar position of the emitters around the VHF gun cathode plug can potentially be exploited for a passive collimation, drastically reducing the amount of transported dark current. Such a scheme is described in more detail in the next section.

If necessary, by moderately reducing the gun operation energy, it is possible to significantly reduce the amount of dark current with a minimal impact on the beam brightness performance. For example, Fig. 11 shows that by reducing the the gun energy from $750 \mathrm{keV}(19.5 \mathrm{MV} / \mathrm{m})$ to $700 \mathrm{keV}$ $(18.2 \mathrm{MV} / \mathrm{m})$, the amount of dark current can be reduced by about a factor 3 and, as simulations have indicated, with a marginal increase of the transverse emittance.

An alternative to the passive collimation is represented by an active sweeper system composed by a transverse kicker followed by a collimator. A possible configuration for such a system is described in Sec. III B.

If a new version of the VHF gun will be built in the future, two additional actions can be undertaken to decrease the amount of dark current. State of the art machining techniques can be used to obtain the best possible finish of the critical area around the cathode plug, and simulations show that a larger radius for the cathode plug area exposed to the rf fields can effectively reduce the amount of transported dark current.

\section{A. Passive removal}

Based on the experimental and simulation results discussed in Sec. II A, the possibility for a passive collimation scheme system to reduce the amount of dark current is studied here. The idea is based on the observation that for a given setting of the solenoids, there could be an optimum position along the beam line where the dark current spots are transversely well separated from the photoemitted beam, as in the case of Fig. 4. By placing a circular collimator (circular aperture) with the proper diameter in such a position, it is possible in principle to remove most of the dark current without affecting the photoemitted beam.

In order to evaluate the feasibility of such a scheme in APEX, a number of simulations were performed using the nominal beam line settings for the emittance compensation. The optimal longitudinal position for the collimator along the beam line is the one where the separation between the main beam and the dark current spots is maximum. For each longitudinal position along the beam line, the minimal acceptable radius for the collimator was assumed to be 3 times the rms transverse size of the photoemitted beam in order to minimize losses of such beam. Simulations using the initial distributions described in Sec. II A allowed us to define the position for the collimator that maximizes the reduction in dark current.

Figure 18 shows the simulated dark current transmission vs the longitudinal position of a collimator of length $1 \mathrm{~cm}$ and radius 3 times the rms transverse beam size of the photoemitted beam at that particular longitudinal position. The cases for charges per bunch of 20,100, and $300 \mathrm{pC}$ are shown. It is worth remarking that at any given longitudinal position, the collimator radii used to obtain the results in the figure were not the same between the different charges. Indeed, the photoemitted beam size depends on the charge per bunch and larger sizes are typically associated with larger charges per bunch. The optimal position for the

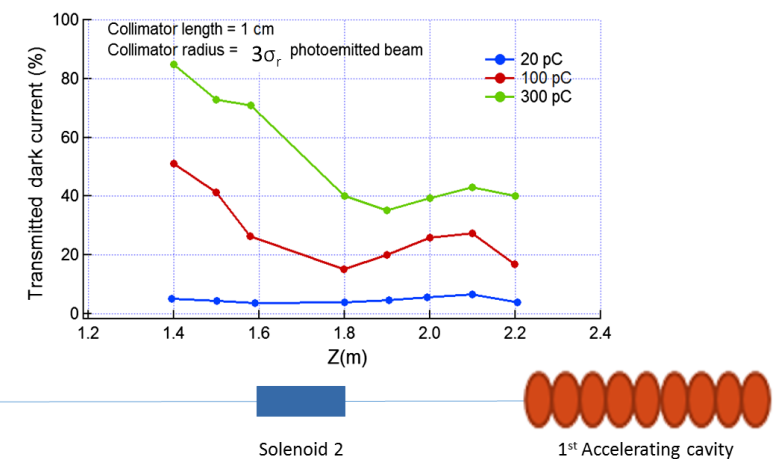

FIG. 18. Dark current transmission vs the longitudinal position of a collimator for three different charges per bunch. The simulations assume a collimator length of $1 \mathrm{~cm}$ and a radius 3 times the rms transverse beam size of the photoemitted beam at that particular longitudinal position. 


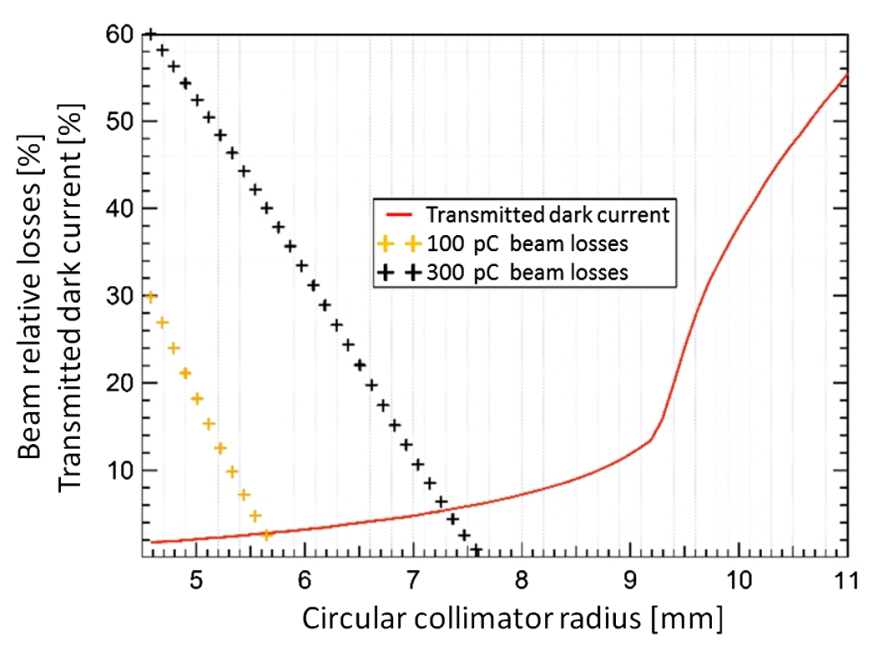

FIG. 19. Beam losses vs circular collimator radius. The collimator used in the simulation has a length of $20 \mathrm{~cm}$ and is located inside solenoid 2. It can be seen that, with a radius of $\sim 8.3 \mathrm{~mm}$, no losses are generated in the 100 and $300 \mathrm{pC}$ (and in the $20 \mathrm{pC}$ not shown in the figure), while $\sim 90 \%$ of the dark current is lost.

collimator is the one where the transmitted dark current has a minimum. From Fig. 18, it can be seen that the region right downstream solenoid 2 represents a good location for a collimator for all the three different charge cases. Additional studies showed that a collimator with $20 \mathrm{~cm}$ length inside solenoid 2 represents a simple and effective solution for all the different charge cases. A summary of the results is reported in Fig. 19 where the relative dark current transmission and the relative beam losses vs the radius of the collimator are shown. One can see from the figure that, for example, with a radius of $8.3 \mathrm{~mm}$ more than $90 \%$ of the dark current is suppressed with no losses for the photoemitted beams for both the 300 and $100 \mathrm{pC}$ case. For the $20 \mathrm{pC}$ case, not shown in the figure, the collimator does not generate losses even for much smaller radii. Figure 19 also shows that in the most challenging case of $300 \mathrm{pC}$ a reasonable orbit stability of a few hundred $\mu \mathrm{m}$ is required to avoid photoemitted beam losses.

The dark current heat load on the collimator is minimal and by using the numbers in Fig. 11 it can be estimated of the order of $1 \mathrm{~W}$. Such a value does not represent a challenge in terms of cooling, but in order to avoid damage to the collimator from the several hundreds of $\mathrm{W}$ if the photoemitted beam is mis-steered, a proper cooling or a fast machine protection system is required.

Calculations and analytical formulas in [24] indicated a negligible emittance increase due to the collimator transverse wakefields if the beam orbit is maintained within $0.5 \mathrm{~mm}$ and/or some longitudinal tapering of the collimator is used.

A possible issue that needs to be further analyzed and investigated is represented by the effects on vacuum and cathode lifetime that scattered shower products induced by the dark current impinging on the collimator can potentially generate.

A test of the described collimation system is planned at APEX.

\section{B. Active removal}

While the nominal APEX repetition rate is $1 \mathrm{MHz}$, the $\mathrm{CW}$ operation naturally implies field emitted bunches at the gun frequency, i.e. $186 \mathrm{MHz}$. Most of the dark current is therefore temporally separated from the photoemitted beam, opening to the possibility of temporal collimation. A fast kicker can be used for this scope by placing it immediately after the VHF gun, where the beam energy and power are lower than in the rest of the injector and a less challenging kicker strength is required.

When the APEX beam line is optimized for a charge per bunch of $300 \mathrm{pC}$, the beam shows a hard edge transverse distribution with $\sim 8 \mathrm{~mm}$ radius at the entrance of the second solenoid. Studies show that in this case, the best possible locations for the kicker and the collimator are in proximity of the two solenoids, with the kicker right after the first solenoid and the collimator $1 \mathrm{~m}$ downstream, before the entrance of the second solenoid. In this configuration, the combination of a circular collimator with radius of $10 \mathrm{~mm}$ and of a kicker strength of $15 \mathrm{mrad}$ shows a potential dark current reduction of $\sim 90 \%$.

Two kinds of kickers have been evaluated, a low frequency resonant kicker and a stripline kicker [25-27]. The resonant kicker, shown in Fig. 20(a), oscillates at $0.5 \mathrm{MHz}$, half of the beam repetition rate. The photoemitted beam crosses the kicker at the "zero-crossing" when the field between the electrodes is zero, and hence its centroid receives no net transverse kick. On the other hand, most of the dark current goes through the kicker when the field is not zero and receives a transverse kick, as shown in Fig. 20(b). It can be calculated that in order to reduce the dark current by $90 \%$, the peak kick has to be $\sim 15 / \sin \left(9^{\circ}\right) \mathrm{mrad}$, i.e. $\sim 96 \mathrm{mrad}$. Such a kick will let $\sim 9$ dark current pulses on each side of the photoemitted beam to "survive."

Because of the finite length of the photoemitted bunch and of the zero-crossing configuration, the particles in the head of the beam will receive a different kick with respect to the ones in the tail. The effect of such a time-dependent kick on the photoemitted beam emittance can be calculated by [28]

$$
\Delta \varepsilon_{n}=k_{r f} \sigma_{z} \sigma_{x} \frac{p_{z} \Delta \theta_{\text {peak }}}{m c}
$$

where $k_{r f}$ is the wave number of the resonant kicker, $\sigma_{x}$ and $\sigma_{z}$ are rms bunch sizes, $p_{z}$ is longitudinal beam momentum, and $\Delta \theta_{\text {peak }}$ is the peak transverse kick of the kicker. For the nominal case of $300 \mathrm{pC}$ and $750 \mathrm{keV}$, we find from simulations $\sigma_{x}=6 \mathrm{~mm}, \sigma_{z}=4.5 \mathrm{~mm}$. The overall 


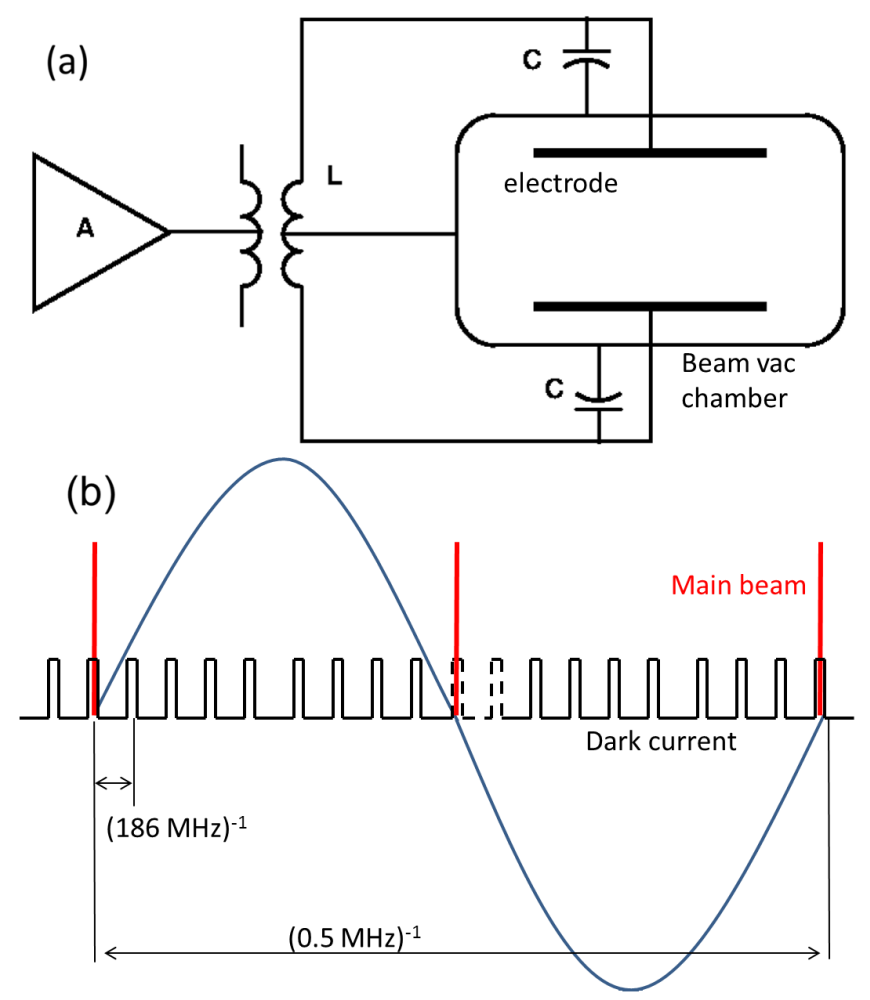

FIG. 20. Schematic diagram of resonant kicker: (a) resonant kicker principle; (b) resonant kicker mode.

emittance growth is less than $10 \%$ of the nominal emittance (0.6 mm mrad).

The advantage of a resonant kicker is that the net kick to the photoemitted beam is zero, so transverse kick uniformity is not an issue. Besides, an rf power supply is generally easier to build than a dc pulser. On the other hand, synchronization is needed between the kicker rf and the beam arrival time. Using low frequencies around $0.5 \mathrm{MHz}$, makes the synchronizing electronics more susceptible to environmental noise, but relaxes the overall requirements on the phase jitter. Simulations show a rms beam divergence at the kicker location of about $\sim 4 \mathrm{mrad}$, and in order to keep the residual kick due to phase jitter at $10 \%$ or lower, the phase jitter at $0.5 \mathrm{MHz}$ should be at or below $0.2^{\circ}$.

The other option is a stripline kicker pulsed at $1 \mathrm{MHz}$. The stripline kicker is superimposed to a dc dipole field, and the two kicks perfectly compensate each other when the kicker is on, allowing the undisturbed passage of the main beam. When the stripline kicker is off, the dark current is swept away by the remaining de dipole field. A pulse length shorter than $100 \mathrm{~ns}$ is needed for dark current reduction of $90 \%$.

Variations of the stripline kick from perfect field flattness will induce emittance growth. A relative time variation of the applied kick voltage of $3 \times 10^{-4}$ within the bunch length would lead to a projected emittance growth of $10 \%$ [0.06 mm mrad, using a similar approach to the one used for Eq. (14)]. Moreover, kick transverse nonuniformity will

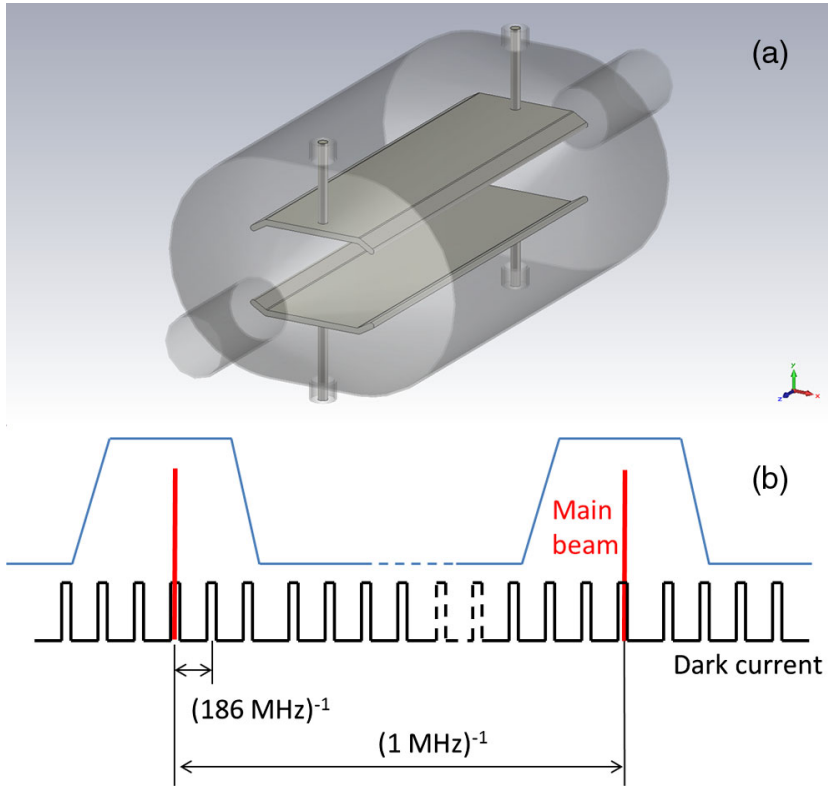

FIG. 21. Schematic diagram of stripline kicker: (a) APEX dark current stripline kicker design based on the ALS camshaft kicker; (b) stripline kicker mode.

induce slice emittance growth, and first-order calculations provide a similar uniformity requirement of about $3 \times 10^{-4}$ rms. Pulse-to-pulse stability will increase the multibunch emittance, leaving residual random kicks on the beam. By applying the same constraint in emittance growth, we find a stability requirement of $2 \%$.

A stripline kicker with the performances outlined above has been designed based on the existing Advanced Light Source (ALS) camshaft kicker [29]. A 3D view of the kicker is shown in Fig. 21(a). In order to achieve the $3 \times$ $10^{-4} \mathrm{rms}$ transverse uniformity requirement, the ALS kicker cross section has been increased by about a factor 3 , and both the chamber and the electrodes shapes have been reoptimized. Because of the lack of beam line space, the stripline length was reduced by about a factor 3 . The kicker plate spacing and length are $4.2 \mathrm{~cm}$ and $20 \mathrm{~cm}$, respectively, and the required voltage on each stripline is $0.8 \mathrm{kV}$ for the $15 \mathrm{mrad}$ kick, a value already within the specification of the ALS camshaft kicker pulser being presently used.

\section{CONCLUSIONS}

The paper presents an extensive characterization in space, intensity, and energy spectrum, of field-emitted electrons from the APEX CW VHF photogun operating at LBNL. The sources of field emission have been localized creating images of the cathode plane with the help of solenoid lenses, and the measurements compared with particle simulations. It was found that, given the peculiar geometry of the cathode plug area, only particles originating from emitters at the plug-cavity interface (on the cavity 
side) have the right net transverse momentum to be transported along the beam pipe (see Fig. 7).

The time-integrated Fowler-Nordheim formula has been used to fit dark current measurements as a function of electric field, extracting the source characteristics. Measurements taken at different times during the gun lifetime suggested a dark current decrease with operations.

Single emitter studies were carried out by taking advantage of the spatial separation between dark current sources. The Fowler-Nordheim plot of a single source was measured, together with its energy distribution. In particular we showed that the spectrum of the single emitter can be used for obtaining the same information on the source found by measuring the emitted current versus the electric field. In Sec. II C 3 a simple mathematical model was derived from the Fowler-Nordheim equation for the instantaneous fieldemitted current, and the results for a particular case reported.

The spatial separation between dark current and photoemitted beam can also be exploited for passive collimation. Such a method was discussed in detail in Sec. III A where it was shown that, by placing a collimator inside the second solenoid and choosing an appropriate diameter, about $90 \%$ of the dark current is removed. Active methods can also be used for dark current mitigation, taking advantage of the different repetition rate of dark current pulses and the main beam, by applying a time-dependent kick. Such methods are generally very suitable for low energy beams, as the needed kick amplitude is small. On the other hand, the tight requirements on the beam emittance directly translates to requirements on kicker performance uniformity and stability. In Sec. III B we discussed two options: a low-frequency resonant kicker and a stripline kicker, defining the tolerances based on an emittance growth smaller than $10 \%$.

The levels of a dark current presented are already within the specifications for the next generation light sources. Nevertheless such values have been obtained without applying any specific surface treatment for dark current reduction. The dry ice cleaning procedure will be tested on the APEX gun, with possible further reduction of produced field-emitted current.

\section{ACKNOWLEDGMENTS}

The authors would like to thank J. Staples for calculating field maps in the cathode region, and Russell Wells for providing the detailed geometry of the cathode area and for stimulating discussions. The authors acknowledge support from DOE Grant No. DE-AC02-05CH11231.

[1] Y. Ding et al. Phys. Rev. Lett. 102, 254801 (2009).

[2] Y. Shen, X. Yang, G. L. Carr, Y. Hidaka, J. B. Murphy, and X. J. Wang, Phys. Rev. Lett. 107, 204801 (2011).

[3] P. Musumeci, J. T. Moody, C. M. Scoby, M. S. Gutierrez, and M. Westfall, Appl. Phys. Lett. 97, 063502 (2010).
[4] X. F. D. Stragier, O. J. Luiten, S. B. van der Geer, M. J. van der Wiel, and G. J.H. Brussaard, J. Appl. Phys. 110, 024910 (2011).

[5] For a review of present technologies see, for example, F. Sannibale, D. Filippetto, and C. Papadopoulos, J. Mod. Opt. 58, 1419 (2011).

[6] R. J. Noer, Ph. Niedermann, N. Sankarraman, and O. Fischer, J. Appl. Phys. 59, 3851 (1986).

[7] R. H. Fowler and L. Nordheim, Proc. R. Soc. A 119, 173 (1928).

[8] D. Filippetto, P. Musumeci, M. Zolotorev, and G. Stupakov, Phys. Rev. ST Accel. Beams 17, 024201 (2014).

[9] J. Staples, F. Sannibale, and S. Virostek, VHF-band Photoinjector, CBP Technical Note 366, LBNL, 2006.

[10] K. Baptiste, J. Corlett, S. Kwiatkowski, S. Lidia, J. Qiang, F. Sannibale, K. Sonnad, J. Staples, S. Virostek, and R. Wells, Nucl. Instrum. Methods Phys. Res., Sect. A 599, 9 (2009).

[11] F. Sannibale et al., Phys. Rev. ST Accel. Beams 15, 103501 (2012).

[12] K. Flottman, http://www.desy.de/ mpyflo/astradokumentation/.

[13] Russell D. Young, Phys. Rev. 113, 110 (1959).

[14] Kwang-Je Kim, Nucl. Instrum. Methods Phys. Res., Sect. A 275, 201 (1989).

[15] Bruce F. Carlsten, Nucl. Instrum. Methods Phys. Res., Sect. A 285, 313 (1989).

[16] C. F. Papadopoulos et al., in Proceedings of FEL2014, Basel, Switzerland (2014), THP057 [http://www.fel2014 .ch/prepress/FEL2014/papers/thp057.pdf].

[17] J. Schmerge et al., in Proceedings of FEL2014, Basel, Switzerland (2014), THP042 (to be published).

[18] R. Latham, High Voltage Vacuum Insulation: Basic Concepts and Technological Practice (Academic Press, New York, 1995).

[19] J. W. Wang and G. A. Loew, in Proceedings of the Joint School RF Engineering for Accelerators (1997) [http://www .slac.stanford.edu/cgi-wrap/getdoc/slac-pub-7684.pdf].

[20] H. Padamsee, and J. Knoblach, AIP Conf. Proc. 474, 212 (1999).

[21] H. Chen et al., Phys. Rev. Lett. 109, 204802 (2012).

[22] A. Brinkmann, D. Reschke, and J. Ziegler, in Proceedings of LINAC08, Victoria, BC, Canada, 2008, p. 803 [https:// accelconf.web.cern.ch/accelconf/LINAC08/papers/thp013 .pdf].

[23] M. Kuriki et al., Proceedings of the 2nd International Particle Accelerator Conference, San Sebastian, Spain (EPS-AG, Spain, 2011), p. 92.

[24] P. Tenenbaum et al., Phys. Rev. ST Accel. Beams 10, 034401 (2007).

[25] J. Fröhlich, Ph.D. thesis, University of Hamburg, DESYThesis-2009-012, 2012.

[26] R. Xiang, A. Arnold, T. Kamps, P. Lu, P. Michel, P. Murcek, H. Vennekate, G. Staats, and J. Teichert, Phys. Rev. ST Accel. Beams 17, 043401 (2014).

[27] J. Staples, CBP Tech Note 429, LBNL, 2013.

[28] Z. Li et al., Proceedings of the 21st Particle Accelerator Conference, Knoxville, TN, 2005 (IEEE, Piscataway, NJ, 2005), p. 2176.

[29] S. Kwiatkowski et al., Proceedings of the 10th European Particle Accelerator Conference, Edinburgh, Scotland, 2006 (EPS-AG, Edinburgh, Scotland, 2006), p. 3547. 